\title{
Diálogos entre a Saúde do Campo e a Saúde Mental: a experiência da Oficina de Educação Popular em Saúde Mental do MST na ESP MG
}

\author{
Bianca Rückert ${ }^{(a)}$ \\ Ana Regina Machado ${ }^{(b)}$ \\ Carine Constancia Alves e Santos ${ }^{(c)}$ \\ Patrícia Cássia Duarte de Brito ${ }^{(\mathrm{d})}$
}

\section{Introdução}

Este artigo apresenta o relato de uma experiência de educação popular em saúde, que vem sendo realizada pela Escola de Saúde Pública do Estado de Minas Gerais - ESP MG e pelo Movimento dos Trabalhadores Rurais Sem Terra - MST. A experiência denominada "Oficina de educação popular em saúde mental para populações assentadas e acampadas em projetos de reforma agrária de Minas Gerais" teve início no ano de 2011, deverá ser concluída até o fim de 2013 e vem sendo realizada em Belo Horizonte e em municípios com assentamentos e acampamentos do MST em Minas Gerais. A ação é destinada às lideranças de saúde do MST e aos trabalhadores das redes públicas de atenção à saúde desses municípios.

Tratando-se de educação popular, a concepção adotada nesta experiência orienta-se por uma abordagem freiriana de educação. Uma perspectiva que reconhece que as organizações populares - enquanto sujeitos políticos em caminhada histórica - produzem saberes a partir dos processos de luta e transformação da realidade social. De acordo com Vasconcelos ${ }^{1}$, no âmbito da saúde, grande parte das experiências de educação popular tem como objetivo construir uma prática de saúde "[...] em que as várias dimensões da doença passam a ser enfrentadas", a partir da superação do fosso cultural existente entre o saber médico, de um lado, e a dinâmica de adoecimento e de cura do mundo popular, de outro.

Nessa perspectiva, a Oficina de Educação Popular em Saúde Mental busca contribuir para a adequação e ampliação das práticas de cuidados em saúde mental, de modo a responder às necessidades de saúde das populações assentadas e acampadas, sobretudo, as relacionadas ao uso abusivo de álcool e outras drogas e ao sofrimento mental grave.

Todo o processo de desenvolvimento da Oficina, desde a sua elaboração à realização das etapas, conta com a participação de militantes do Setor Saúde do MST de Minas Gerais e trabalhadores de Saúde Mental da ESP MG. A escolha do referencial da Educação Popular tem possibilitado o diálogo entre os saberes da Saúde do Campo e os saberes (a) Movimento dos Trabalhadores Rurais Sem Terra; Núcleo de Redes de Atenção à Saúde. Escola de Saúde Pública do Estado de Minas Gerais. Rua Uberaba, 780, Barro Preto, Belo Horizonte, MG, Brasil. 30180-080. biaruckert@yahoo.com.br

(b,d) Núcleo de Redes de Atenção à Saúde. Escola de Saúde Pública do Estado de Minas Gerais. Belo Horizonte, MG, Brasil. anarmachado@uol.com.br padu@hotmail.com

(c) Movimento dos Trabalhadores Rurais Sem Terra. Belo

Horizonte, MG, Brasil. caninanas@yahoo.com.br 
da Saúde Mental, na busca pela produção de novas compreensões sobre o sofrimento mental e o uso nocivo de álcool e outras drogas e na valorização das práticas de cuidado e formas de luta por saúde de todos os envolvidos: lideranças de saúde e demais integrantes do MST, trabalhadores da educação, da atenção e da gestão do Sistema Único de Saúde - SUS.

Este artigo consiste em um relato de experiência em desenvolvimento, que buscou enfatizar os diálogos e as trocas efetivadas entre sujeitos, instituições, saberes e práticas na produção de uma ação de educação popular em saúde. O texto apresenta os antecedentes da construção da Oficina, seu projeto político-pedagógico, os primeiros resultados dessas trocas e perspectivas de continuidade da ação.

\section{A Conquista da Oficina}

A Oficina de Educação Popular em Saúde Mental teve origem em uma jornada de lutas do MST MG, em abril de 2011, por ocasião do III Encontro dos Movimentos Sociais Mineiros. Nesta, o Movimento apresentou ao Governo do Estado uma pauta reivindicatória para seus assentamentos e acampamentos de reforma agrária.

As mobilizações, marchas, ocupações de terras e órgãos públicos são uma das expressões mais fortes do MST, marcando sua trajetória ao longo dos seus quase trinta anos de existência. A luta pela saúde como direito integra essa agenda e busca denunciar as iniquidades em saúde vividas pelas populações do campo, bem como valorizar as culturas, os saberes e as potencialidades dessas populações. É importante considerar que, no campo e na floresta, especialmente, as populações da reforma agrária, agricultores familiares, comunidades indígenas, quilombolas e extrativistas, entre outras, encontram vários obstáculos ao acesso às políticas públicas de saúde e todas as demais que garantam as condições de vida e os direitos necessários à permanência nesses territórios ${ }^{(\mathrm{e})}$. Tais obstáculos são ainda maiores no que se refere à Saúde Mental, por conta dos desafios da implantação dos serviços substitutivos nos municípios de pequeno porte ${ }^{(f)}$. Esta experiência insere-se, portanto, em um contexto de luta por políticas públicas de promoção de equidade em saúde dessas populações.

$\mathrm{Na}$ ocasião, o Setor de Saúde em Minas Gerais(g) já percebia a necessidade da problematização da temática da Saúde Mental. Embora não houvesse informações na literatura que corroborassem tal necessidade ${ }^{(h)}$, constatava-se que alguns problemas relacionados ao sofrimento mental haviam se transformado em temas recorrentes nas discussões dos assentamentos e acampamentos organizados pelo MST, sendo notório o aumento do consumo prejudicial de álcool e, até mesmo, outras drogas. As demandas relacionadas ao sofrimento mental, desde transtornos leves aos mais graves, também se apresentavam como motivos de preocupação deste grupo populacional. Estas, em grande medida relacionadas às dificuldades de acesso aos serviços de saúde ou despreparo na abordagem e na condução dos cuidados estabelecidos. A realidade da saúde no campo revela que muitos casos que poderiam ser rapidamente orientados e conduzidos tornam-se crônicos, trazendo prejuízos não somente no que tange aos prognósticos, como também onerando social e economicamente todos os envolvidos. O Movimento incluiu, então, o tema na sua luta pelo direito à saúde. A expectativa era possibilitar, por meio de uma ação de formação, a construção de novos olhares e significados para o sofrimento (e) A recente publicação da Política Nacional de Saúde Integral das Populações do Campo e da Floresta - PNSIPCF reitera esta afirmativa. (f) Importante considerar que o acesso à Rede de Atenção Psicossocial por parte das populações do Campo e da Floresta é um dos mecanismos explicitados no Eixo 1 da PNSIPCF.

(g) O setor saúde é uma das instâncias organizativas no MST. Neste, os/ as trabalhadores/as pertencentes às áreas de acampamento e assentamentos se organizam em instâncias núcleo de base de famílias, coordenação de área, coordenação regional, coordenação e direção estaduais, coordenação e direção nacional. De forma transversal às instâncias, organizam-se os setores e coletivos, como Saúde, Educação, Produção

Comunicação, Frente de Massa, Direitos Humanos, Juventude, Gênero, Cultura e Finanças, entre outros, para a realização de tarefas e discussões específicas. Estes também se organizam desde o nível local, regional, estadual e nacional.

(h) Não foram encontrados estudos que permitissem dimensionar e caracterizar a ocorrência do consumo de drogas e de problemas de saúde mental em populações assentadas e acampadas em Minas Gerais. Apenas foi identificado um estudo, realizado por Faria et al2 junto a trabalhadores rurais da região serrana do Rio Grande do Sul, que constatou a ocorrência de alcoolismo em índice semelhante $(7 \%)$ a outros encontrados em estudos com populações urbanas. Constatou-se, também, a ocorrência de transtornos psiquiátricos menores em índice superior $(38,6 \%)$ ao encontrado em um estudo realizado na população urbana de Pelotas $(21,5 \%)$, também no Rio Grande do Sul. 
mental, a loucura e o uso nocivo de álcool e outras drogas, bem como de práticas de promoção e de cuidado orientadas por um conceito ampliado de saúde, pelas contribuições da Reforma Psiquiátrica e da Redução de Danos, ou seja, a partir de dois referenciais do campo da Saúde Mental no Brasil.

Para responder à solicitação do MST, o Governo do Estado de Minas Gerais, por meio da Secretaria Estadual de Saúde (SES), criou um grupo de trabalho que foi encarregado de apresentar uma proposta de ação educativa. Este contou com representantes da própria SES, trabalhadores da ESP MG e representantes do Setor Saúde do MST MG.

\section{A construção da proposta da Oficina}

A construção da proposta da Oficina ocorreu entre agosto e dezembro de 2011, em uma série de encontros que permitiram um diálogo entre os saberes e fazeres das lideranças de saúde do MST e dos trabalhadores de saúde mental da ESP MG ${ }^{(i)}$.

O Setor de Saúde do MST MG já tinha clareza quanto à adoção do referencial da Educação Popular em Saúde nesse processo, uma vez que já buscava o desenvolvimento de práticas orientadas por esta concepção em experiências envolvendo práticas populares de cuidado, saúde ambiental, segurança alimentar e nutricional, entre outras. Estas, entretanto, dificilmente produziam alguma interlocução com os trabalhadores do SUS e mobilizavam, geralmente, apenas os recursos comunitários.

Os trabalhadores de saúde mental da ESP MG, embora não tivessem clareza do referencial adotado, possuíam um histórico de atuação junto a movimentos sociais, como a Luta Antimanicomial e o Movimento Estudantil da área de saúde. Assim como estes, apresentavam um compromisso com a luta pelo direito à saúde, pela Reforma Psiquiátrica e com práticas de atenção a usuários de drogas pautadas pelos princípios da Redução de Danos.

$\mathrm{Na}$ construção desta experiência, destacam-se algumas contribuições da Reforma Psiquiátrica, como a promoção do cuidado em liberdade; o respeito ao diferente e à singularidade; a utilização de diversos saberes na compreensão dos sofrimentos mentais e nas práticas de cuidados; o reconhecimento de que a doença mental não traduz a experiência de vida das pessoas com sofrimento mental grave; a importância da produção de diferentes laços e inserções sociais, da promoção de novas identidades e da ampliação da autonomia das pessoas com sofrimento mental grave.

No que tange à Redução de Danos, é importante destacar as contribuições desta abordagem na promoção da cidadania dos usuários e no redimensionamento das práticas de cuidados. Este novo olhar valoriza diferentes estratégias e possibilidades relacionadas à diminuição dos sofrimentos e danos associados ao consumo de drogas e ao fortalecimento das redes de apoio social.

A equipe formada reconhecia, então, a importância de construir uma ação de formação que adotasse o conceito ampliado de saúde e valorizasse as lutas do MST - dentre estas, a luta pelo direito à saúde - bem como o aprendizado a partir de outras experiências e movimentos sociais. Nesse processo, os participantes do Grupo de Trabalho compartilharam princípios e estabeleceram diálogos sempre pautados pelo que Stotz ${ }^{3}$ considera como traço fundamental da Educação Popular em Saúde, o "[...] reconhecimento, palavra que tem o sentido de admitir um outro saber, tão válido no âmbito do diálogo quanto o saber técnico-científico". Mais do que admitir outros saberes, os participantes do Grupo foram permeáveis e 
permitiram se modificar pelo diálogo. Esta permeabilidade pode ser percebida no Projeto Político-Pedagógico da Oficina, nas atividades realizadas e aprendizados decorrentes desta experiência.

\section{O Projeto Político-Pedagógico}

A educação popular, enquanto concepção orientadora desta experiência, se expressa na proposição do diálogo entre saberes e práticas da Saúde Mental, da Saúde do Campo e na articulação dos diversos atores em torno dessas temáticas - movimentos sociais, instituição de ensino, gestão e trabalhadores dos serviços de saúde.

A proposta consiste na realização de oficinas sobre Saúde Mental e Saúde do Campo e abrange duas turmas, cada uma com cerca de 70 participantes - 35 lideranças de saúde do MST e 35 trabalhadores da atenção primária e saúde mental da rede pública de saúde dos municípios envolvidos no projeto. A Oficina envolve três etapas presenciais, realizadas na ESP MG, e uma etapa local, realizada nas áreas de reforma agrária.

A primeira etapa teve como público-alvo as lideranças de saúde do $M_{S T}{ }^{(j)}$ e a duração de quatro dias. Para a segunda etapa espera-se a participação dos trabalhadores do SUS e alguns representantes do Setor de Saúde do MST. Essas etapas pretendem desenvolver uma ampliação dos olhares sobre as temáticas da Saúde do Campo, Reforma Psiquiátrica e Redução de Danos no cuidado aos usuários de álcool e outras drogas. A partir desses pressupostos, a terceira etapa consistirá em um momento de integração entre os públicos participantes, visando à construção de estratégias compartilhadas de cuidado em saúde mental e qualificação dos serviços prestados no âmbito dos territórios envolvidos na experiência.

Entre as etapas presenciais realizam-se as locais, um processo fundamentado na construção de vínculos entre prática e teorização, comunidade e escola ${ }^{(k)}$, ensino e serviço(l), por meio de ferramentas e metodologias dialógicas entre tempos, espaços e saberes, em um movimento de busca da práxis político-pedagógica. Para os participantes da primeira etapa, ou seja, as lideranças do MST, esse momento consiste na realização de oficinas nos assentamentos e acampamentos e no mapeamento de dados sobre a situação de acesso aos serviços de saúde - em especial à rede de saúde mental -, os principais problemas de saúde enfrentados por esse grupo populacional e práticas de saúde realizadas no âmbito comunitário. Esse processo se insere em uma estratégia pedagógica que pretende facilitar as discussões, qualificar a percepção e a análise em saúde em um nível local. As atividades locais contam com a contribuição das referências de saúde das regiões envolvidas, que atuam como Tutoras de Dispersão. Para os participantes da segunda etapa, ou seja, os trabalhadores do SUS, esse momento consistirá na visita a uma área de reforma agrária e no mapeamento do território, no tocante aos recursos terapêuticos e fluxos de atenção em saúde mental. Ao final da experiência, propõe-se a elaboração de uma cartilha que apresentará uma linha de cuidados em saúde mental, envolvendo as práticas dos assentamentos e acampamentos e as práticas dos serviços de saúde.

A avaliação da Oficina é planejada a partir dos princípios da Avaliação Formativa, o que implica compreendê-la como integrante do processo de ensino-aprendizagem. Nessa perspectiva, ao mesmo tempo em que se processa a atividade formativa, (i) É importante mencionar que, assim como nas demais atividades do Setor de Saúde do MST, o público é majoritariamente feminino, o que deve ser compreendido à luz do referencial da divisão sexual do trabalho, uma vez que o trabalho de saúde e de cuidados é uma prática historicamente atribuída às mulheres. (k) De acordo com a Pedagogia da Alternância, uma das matrizes que integra a Educação do Campo.

(I) Conforme proposições do Currículo Integrado, uma das matrizes que integra a Educação Permanente em Saúde. 
analisa-se sua contribuição para a formação de uma visão crítica sobre a realidade vivida e a transformação das práticas de todos os envolvidos.

\section{A Oficina como espaço de diálogo}

Considera-se que a primeira etapa da Oficina cumpriu o papel de revisão de conceitos, paradigmas e valores em relação às temáticas trabalhadas. A construção desse enfoque foi possível, na medida em que esta etapa constituiu-se em tempo/espaço de problematização, vivência de valores e práticas e socialização, apreensão e produção de saberes.

Dentre as diversas intencionalidades presentes na experiência, é importante destacar, ainda, a perspectiva feminista, dado o perfil das lideranças de saúde e a forte articulação entre a luta por saúde e a luta das mulheres no MST MG. Assim, a temática "A Saúde das Mulheres do Campo" foi abordada já no primeiro dia da Etapa e proporcionou a problematização da realidade vivida a partir da ótica feminista. Esse tema permitiu reflexões sobre as condições de vida e trabalho das mulheres no campo, a sobrecarga de trabalho e seus impactos no processo de adoecimento dessas mulheres. Uma sobrecarga que se refere não somente à histórica e injusta divisão sexual do trabalho, mas, também, à ausência do Estado em garantir políticas sociais para o campo. A compreensão desse contexto foi importante para se abordar a medicalização do corpo e da vida das mulheres camponesas e questionar os interesses com os quais esta conduta se compromete, assim como seu impacto no sofrimento e na diminuição da autonomia, da autoestima e do protagonismo dessas mulheres.

Em seguida, a roda de conversa "Concepção e Experiências de Saúde do MST" possibilitou o diálogo sobre o processo de saúde-doença-cuidado e a discussão acerca dos determinantes sociais da saúde no campo e do papel das práticas populares de cuidado. Essa discussão levantou aspectos significativos que integram o 'Conceito de Saúde do MST' - dentre eles, terra, trabalho, cuidado com o ambiente, segurança alimentar, lutas, organização política e valores como cuidado e solidariedade.

Nesta experiência, a problematização da realidade proporcionou um terreno fértil para se discutir a saúde mental a partir do conceito ampliado de saúde, bem como compreender a dimensão social inscrita no sofrimento mental. Stotz et al ${ }^{4}$ reconhecem a problematização como uma ferramenta que permite partir de leituras mais amplas e superficiais da realidade para compreender os pontos-chaves - que, nesse caso, estão relacionados ao processo saúde-doença-cuidado. Tais autores afirmam, ainda, que:

A concepção transformadora da educação parte das condições concretas de vida para estabelecer, pelo diálogo, processos educativos capazes de melhorar sua vida. A problematização é uma forma de explicar uma situação e, também, uma escolha pedagógica na qual a participação de todos é estimulada, em todos os momentos. Ao mesmo tempo em que trazem suas ideias - ensinam - as pessoas aprendem. E ao aprenderem, crescem e se tornam mais fortes ${ }^{4}$.

Na perspectiva de propiciar o diálogo entre as diversas experiências e saberes das lutas pelo direito à saúde e das práticas de cuidado, foram convidados movimentos sociais, grupos culturais e trabalhadores de saúde. Destes, destaca-se a participação do grupo "Meninas de Sinhá", constituído por mulheres moradoras da periferia de Belo Horizonte, que em diversificados espaços sociais cantam com alegria, contam suas histórias de vida e encantam com cirandas e brincadeiras de roda. Outro grupo, o "Xicas da Silva", através de representantes, também encantou com sua intervenção de cenopoesia. O contato com essas experiências, somado a atividades como roda de contar histórias, sarau e oficina de teatro, propiciou a sensibilização das educandas sobre a importância e o reconhecimento da cultura popular como estratégia de promoção da saúde.

A participação de organizações, como Associação dos Usuários do Serviço de Saúde Mental de Minas Gerais, Movimento Nacional de População de Rua, Pastoral de Rua, entidade Redutora de Danos e trabalhadores de saúde coletiva e de saúde mental com trajetória no SUS, articulada ao recurso da dramatização sobre as situações vividas nos assentamentos e acampamentos, permitiu a aproximação desses sujeitos a partir das experiências do sofrimento mental grave e do uso abusivo de drogas. Tais atividades 
possibilitaram aprendizados significativos, como a importância do respeito, do diálogo, do cuidado e da afetividade, e a construção de novos valores em relação às pessoas com sofrimento mental grave ou em uso prejudicial de álcool e outras drogas.

O relato de um usuário de drogas e morador de rua sobre os cuidados recebidos - que não se pautavam pela abstinência por parte dos cuidadores - e as melhoras que obteve - ainda que não conseguisse interromper o uso das drogas propiciou a reflexão sobre a abordagem da redução de danos como possibilidade de se diminuir o impacto negativo que o uso nocivo de drogas pode gerar na vida das pessoas. A experiência apresentada permitiu a reflexão sobre os motivos, os contextos de vida, as dificuldades encontradas no cotidiano, os fatores que ajudam e atrapalham na abordagem, a partir do olhar do próprio sujeito. Outro relato marcante foi desenvolvido por usuários de saúde mental sobre a participação no Movimento da Luta Antimanicomial, a construção de novos laços sociais e a ressignificação dos valores e da própria vida.

Esses relatos permitiram evocar lembranças de situações vividas e propiciaram reflexões sobre as maneiras como os acampamentos e os assentamentos lidam com as pessoas em sofrimento mental grave ou em uso prejudicial de álcool e outras drogas. Tais reflexões permitiram compreender a importância da construção de novos valores, não mais excludentes e moralistas, assim como os valores sociais hegemônicos, dos quais os Sem Terra já foram vítimas inúmeras vezes.

Nesse sentido, foi possível reconhecer a dimensão cuidadora e formadora que o movimento social desempenha na vida das pessoas excluídas socialmente. Os depoimentos das lideranças de saúde do MST mostraram que a inserção na luta pela terra, inicialmente por uma questão econômica, assume outras dimensões na vida desses sujeitos, gerando espaços de participação e trabalho e, consequentemente, construção de identidades, sociabilidades e autonomia. O MST foi citado como movimento social que ajuda a construir e a reconstruir a humanidade nas pessoas, importante contribuição da Pedagogia do Movimento ${ }^{5}$ ao cuidado em saúde mental e à promoção da saúde, que surge como uma das respostas aos questionamentos das lideranças acerca da abordagem aos usuários de álcool e outras drogas.

A Pedagogia do Movimento, segundo Caldart ${ }^{5}$, reconhece que os seres humanos gestam uma pedagogia na própria dinâmica das lutas sociais, ao assumirem coletivamente a condição de sujeitos de seu destino, social e humano. A humanização como projeto da luta do MST se aproxima, assim, do projeto da Reforma Psiquiátrica e da Redução de Danos. Aqui, os depoimentos sobre as trajetórias de vida, histórias de lutas, violências vividas, conquistas e crescimentos ganham destaque e geram o compartilhamento de compreensões sobre o sofrimento mental e sua relação com a dinâmica de vida dos/as trabalhadores/as, bem como a contribuição dos movimentos sociais e suas redes de apoio social no fortalecimento da autoestima, autonomia e dignidade.

Nessa mesma perspectiva, foi reconhecido o potencial do cuidado, das práticas populares de saúde e das práticas integrativas e complementares desenvolvidas no MST, que muitas vezes envolvem a terapia floral, a fitoterapia, a radiestesia, o passe espiritual, o reiki, entre outras ${ }^{(m)}$. A valorização e a socialização desses saberes na I Etapa foram possíveis por meio das oficinas práticas que foram conduzidas pelas lideranças do MST, das rodas de conversas e, sobretudo, a partir das trocas efetivadas no próprio processo de socialização entre as participantes.

A temática do direito ao SUS, por sua vez, apresentou-se como objeto de inquietação e questionamento entre as lideranças de saúde. Como garantir o direito ao SUS para as populações do campo? Como assegurar que os princípios

(m) Cabe aqui destacar que algumas destas integram a Política Nacional de Práticas Integrativas e Complementares no SUS. 
da Reforma Sanitária e da Reforma Psiquiátrica estejam presentes, de fato, na realidade dessas populações? Estas e outras questões foram apresentadas diante da complexidade inerente ao tema da Saúde Mental e do direito ao SUS no contexto da reforma agrária.

As vivências relatadas embasaram a construção de propostas de trabalho para a etapa local da experiência, a serem desenvolvidas pelos coletivos regionais de saúde do MST em suas respectivas regiões. Até então, as oficinas realizadas nos assentamentos e acampamentos foram planejadas a partir de cada contexto, com respeito e valorização dos processos organizativos locais. Para tanto, consideram-se aspectos como histórico de organização da saúde na região, principais demandas locais, recursos disponíveis, contexto político, entre outros.

É importante destacar a capilaridade desta experiência em nível estadual e a possibilidade dela oferecer um panorama da consolidação da Reforma Psiquiátrica nos municípios envolvidos, o que pode contribuir no avanço da Política de Saúde Mental para além das áreas do MST. Os relatos das lideranças sobre as tentativas de acesso à atenção em Saúde Mental mostram que ainda são muitos os desafios à efetivação de uma rede de serviços substitutivos que atenda às populações assentadas e acampadas. Os questionamentos das lideranças sobre as estratégias de luta para a garantia de direitos e consolidação dos princípios da Reforma Sanitária e Reforma Psiquiátrica no campo mostram-se, assim, extremamente pertinentes. Com isso, esta experiência pode apresentar um potencial disparador de processos reivindicatórios, à medida que amplia a compreensão em Saúde Mental e permite visualizar as lacunas existentes na Rede de Atenção.

No que tange à construção de linhas de cuidado em Saúde Mental para o campo, os saberes e valores no âmbito das práticas populares de cuidado desenvolvidas pelo MST parecem propiciar um substrato significativo para o início desta construção. Este é um elemento da proposta pedagógica que se fortalece na etapa local da Oficina, uma vez que essas práticas se sobressaem nos relatos de experiências ${ }^{6}$ e nos princípios e objetivos ${ }^{7}$ da saúde do Movimento.

De acordo com Vasconcelos ${ }^{1}$, a articulação dessas práticas ao SUS consiste em uma das grandes contribuições da Educação Popular em Saúde à Reforma Sanitária, ao reconhecer que esta luta não se resume à expansão do direito à assistência à saúde, mas abrange, também, o tipo e a qualidade dos serviços oferecidos. Além disso, vai ao encontro das diretrizes da Política Nacional de Saúde Integral das Populações do Campo e da Floresta, que reconhece as contribuições das práticas e saberes dessas populações ao SUS.

Nesta experiência, a ESP MG se aproxima da realidade dos assentamentos e acampamentos, conhece algumas das dificuldades desse grupo populacional na garantia do direito à saúde e, a partir disso, participa do desafio de construção compartilhada de cuidados em saúde mental. Nessa perspectiva, contribui para dar visibilidade e valorizar as estratégias já adotadas pelo MST, seja na produção de cuidados, seja na promoção da saúde.

É importante considerar, ainda, que a presença do MST na Escola gerou grande repercussão. A presença marcante dos símbolos, cantos e mística do Movimento foi acolhida de maneira respeitosa e solidária. Vários trabalhadores da Escola, mesmo os não envolvidos diretamente na ação, dispuseram-se a contribuir na Ciranda Infantil, montada para acolher os filhos das participantes da Oficina. Nesse momento, foi perceptível a contribuição da comunidade escolar com a ação educativa, as demandas e as propostas de mudanças a ela associadas.

Sem dúvida, esta experiência serviu para reforçar e atualizar o compromisso da Escola com uma formação transformadora e voltada para a conquista do direito à saúde. Somado a isto, esta experiência tem se tornado uma referência para o Núcleo de Redes de Atenção à Saúde, que vislumbra a possibilidade de outros trabalhos com concepções afins. Agrega, portanto, novos valores às práticas formativas, coerentes com princípios da educação permanente e da educação popular em saúde.

\section{Algumas considerações}


O primeiro aspecto a ser destacado a respeito dos diálogos que se efetivaram entre o Setor de Saúde do MST e os trabalhadores da ESP MG é que essa construção foi possível a partir de algumas compreensões compartilhadas por ambos os atores, destacando o respeito e a valorização dos saberes e práticas de cuidados já desenvolvidas pelo MST; a importância da construção conjunta de novas formas de cuidados, envolvendo movimento social e trabalhadores de saúde, a partir da contribuição dos diferentes saberes; o reconhecimento de que a saúde não se alcança exclusivamente por meio da aplicação de saberes técnico-científicos; a compreensão de que a luta pela terra e pela saúde empreendida pelo MST se aproxima da luta antimanicomial e da luta dos profissionais de saúde por um sistema de saúde mais justo, solidário e resolutivo; e a importância dos movimentos sociais no avanço e na implementação de políticas sociais.

A partir dessas compreensões, a Saúde Mental constituiu-se em tema gerador que permitiu a problematização e o diálogo acerca do conceito de saúde, de promoção, de atenção e da luta pela garantia de direitos. Por outro lado, contribuiu para a ressignificação dos valores do cuidado no MST, aproximando-o de valores mais humanos e solidários.

Com isso, já é possível o delineamento de algumas linhas de cuidado em saúde mental que podem ser desenvolvidas junto às populações assentadas e acampadas, tais como a valorização das práticas populares de cuidado, dos saberes locais, da autoestima e da identidade como potencial gerador de saúde; o fortalecimento do acesso à rede de serviços substitutivos em saúde mental; a reflexão coletiva sobre o sofrimento mental e o abuso de álcool e outras drogas e sobre as dimensões social e subjetiva inscritas nesses fenômenos; o reconhecimento da escuta, do diálogo e do cuidado como gerador de autonomia, sem desconsiderar a responsabilização dos usuários; a adoção de diversas estratégias de promoção da saúde e reabilitação psicossocial, entre as quais se incluem a produção cultural, o trabalho e a inserção no movimento social.

Dessa forma, esta experiência mostra o quão fecundo pode ser o diálogo entre a Saúde do Campo e a Saúde Mental, orientado pelos princípios da educação popular em saúde. Nessa caminhada, a educação popular marca presença como instrumento capaz de promover o diálogo entre as experiências dos movimentos sociais, da instituição formadora, da atenção e da gestão, entre outros atores, ao valorizar os diferentes saberes e propor ações integradoras e mais adequadas à realidade de saúde das populações. Mostra-se, portanto, como um saber que orienta os difíceis caminhos da Saúde do Campo, do cuidado na Saúde Mental, da Educação Permanente, das lutas sociais.

\section{Referências}

1. Vasconcelos $E M$. Redefinindo as práticas de saúde a partir da educação popular nos serviços de saúde. Interface (Botucatu). 2001;5(8):121-1. http://dx.doi.org/10.1590/S1414-32832001000100009

2. Faria NMX, Facchini LA, Fassa AG, Tomasi E. Processo de produção rural e saúde na serra gaúcha: um estudo descritivo. Cad Saúde Pública. 2000;16(1):115-28. http://dx.doi.org/10.1590/S0102-311X2000000100012

3. Stotz EN. Enfoques sobre educação popular e saúde. In: Ministério da Saúde, Secretaria de Gestão Estratégica e Participativa, Departamento de Apoio à Gestão Participativa. Caderno de educação popular e saúde. Brasília: Ministério da Saúde; 2007. p. 46-57.

4. Stotz EN et al. Educação popular em saúde. In: Martins $C M$, Stauffer $A B$, organizadores. Educação e saúde. Rio de Janeiro: Fiocruz; 2007. p. 35-70.

5. Caldart RS. Pedagogia do Movimento Sem Terra. 3a ed. São Paulo: Expressão Popular; 2004.

6. Movimento dos Trabalhadores Rurais sem Terra. Relatos de Experiências em Saúde. Brasília, DF: Movimento dos Trabalhadores Rurais sem Terra; 2005.

7. Movimento dos Trabalhadores Rurais sem Terra. Coletivo Nacional de Saúde. Boletim Informativo; 2007. 
O artigo apresenta reflexões a partir da experiência da Oficina de Educação Popular em Saúde Mental, realizada pela Escola de Saúde Pública do Estado de Minas Gerais e pelo Movimento dos Trabalhadores Rurais Sem Terra. A partir do registro da experiência, buscou-se enfatizar os diálogos e as trocas efetivadas entre os sujeitos, as instituições, os saberes e as práticas de saúde. As reflexões apontam para as contribuições da educação popular em saúde no diálogo entre campos de saberes e práticas na construção de linhas de cuidado em saúde mental que envolvam os assentamentos e acampamentos do MST e o Sistema Único de Saúde.

Palavras-chave: Educação Popular em Saúde. Diálogo. Práticas de Saúde. Saúde do Campo. Saúde Mental.

Dialogues between the Field Health and the Mental Health: the experience of the "Popular Education on Mental Health of MST Workshop" in ESP MG

The present paper reflects about the experience of the Popular Education on Mental Health Workshop, held by the School of Public Health of the State of Minas Gerais and the Movement of Landless Rural Workers. From the record of the experiment, we tried to emphasize the dialogues and the exchanges that were effectives between individuals, institutions, knowledge and health practices. The reflections pointed to the contributions of popular education in health through the dialogue between fields of knowledge and practice, building lines of mental health care involving the settlements and camps of the MST and the Unified Health System.

Keywords: Popular Education in Health. Dialogue. Health Practices. Health Field. Mental Health.

\section{Diálogos entre la Salud en el Campo y la Salud Mental: la experiencia del Taller de Educación Popular en Salud Mental DEL MST en la ESP MG}

El artículo presenta reflexiones a partir de la experiencia del Taller de Educación Popular en Salud Mental, realizado por la Escuela de Salud Pública del Estado de Minas Gerais y por el Movimiento de los Trabajadores Rurales Sin Tierra. A partir del registro de la experiencia, se buscó enfatizar los diálogos y los intercambios efectivos entre los sujetos, las instituciones, los conocimientos y las prácticas de salud. Las reflexiones apuntan los aportes de la educación popular en salud, en el diálogo entre el campo del conocimiento y la práctica en la construcción de líneas de cuidado en salud mental que involucren los asentamientos y campamentos del MST y el Sistema Único de Salud.

Palabras claves: Educación Popular de la Salud. Diálogo. Práticas de Salud. Salud en el Campo. Salud Mental. 
